\title{
Integral Inequality of Hardy-Type on Time Scales
}

\author{
Emmanuella Ehui Aribike1, Yisa Oluwatoyin Anthonio', Kamilu Rauf2 ${ }^{*}$, Michael Oyelami Ajisope ${ }^{2}$ \\ ${ }^{1}$ Department of Mathematics and Statistics, Lagos State Polytechnic, Ikorodu, Nigeria \\ ${ }^{2}$ Department of Mathematics, University of Ilorin, Ilorin, Nigeria \\ Email: aribike.ella@yahoo.com, anthonioii@yahoo.com, ${ }^{\star}$ krauf@unilorin.edu.ng, majisope@gmail.com
}

How to cite this paper: Aribike, E.E., Anthonio, Y.O., Rauf, K. and Ajisope, M.O. (2019) Integral Inequality of Hardy-Type on Time Scales. Advances in Pure Mathematics, 9, 534-543.

https://doi.org/10.4236/apm.2019.96025

Received: March 4, 2019

Accepted: June 25, 2019

Published: June 28, 2019

Copyright (c) 2019 by author(s) and Scientific Research Publishing Inc. This work is licensed under the Creative Commons Attribution International License (CC BY 4.0).

http://creativecommons.org/licenses/by/4.0/

\begin{abstract}
Inequalities are essential in the study of Mathematics and are useful tools in the theory of analysis. They have been playing a critical role in the study of the existence and uniqueness properties of solutions of initial and boundary value problems for differential equations as well as difference equations with their bounds. In this paper, we obtain new integral inequalities mainly by using some known inequalities. Various generalizations of Hardy's inequality are special cases of the results therein.
\end{abstract}

\section{Keywords}

Time Scale, Measurable, Convex Functions, Hardy-Type Inequalities

\section{Introduction}

Some inequality were in their reigns such as Wirtinger's, Holder's, Cauchy's, Minkwoski's, Hardy's and Opial's inequalities. Opial [1] established an inequality involving integral of a function and it's derivatives as follows:

Theorem 1.1. Let $\eta(\sigma) \in C^{\prime}[0, \lambda]$ be such that $\eta(0)=\eta(\lambda)=0$, and $\eta(\sigma)>(0, \lambda)$. Then, the following inequality holds.

$$
\int_{0}^{\lambda}\left|\eta(\sigma) \eta^{\prime}(\sigma)\right| \mathrm{d} \sigma \leq \frac{\lambda}{4} \int_{0}^{\lambda}\left(\eta^{\prime}(\sigma)\right)^{2} \mathrm{~d} \sigma
$$

in (1.2), the constant $\frac{\lambda}{4}$ is the best possible.

This inequality and it's generalisations have various applications in the theories of differential and difference equations.

In [2], Olech simplified the proof and noted that the positive requirement of $\eta(\sigma)$ in (1.2) is unnecessary and that inequality (1.2) holds absolutely continuous in $[0, \lambda]$. He however, demonstrated the following result: 
Theorem 1.3. Let $\eta(\gamma)$ be absolutely continuous in $[0, \rho]$ such that $\eta(0)=\eta(\rho)=0$ and $\eta(\gamma)>0$ in $(0, \rho)$ with $\eta(\gamma) \in C^{\prime}[0, \rho]$. Then the following inequality holds.

$$
\int_{0}^{\rho} \eta(\gamma) \eta^{\prime}(\gamma) \mathrm{d} \gamma \leq \frac{\rho}{2} \int_{0}^{\rho}\left(\eta^{\prime}(\gamma)\right)^{2} \mathrm{~d} \gamma
$$

In [3], Hardy obtained the following result:

Theorem 1.5. If $\lambda>1, \rho \geq 0$, and $\varpi(\xi)=\int_{0}^{x} \rho(\xi) \mathrm{d} \xi$, then

$$
\int_{0}^{+\infty}\left(\frac{\varpi(x)}{x}\right)^{\lambda} \mathrm{d} x<\left(\frac{\lambda}{\lambda-1}\right)^{\lambda} \int_{0}^{+\infty} \rho^{\lambda}(x) \mathrm{d} x
$$

unless $\rho \equiv 0$. The constant $\left(\frac{\lambda}{\lambda-1}\right)^{\lambda}$ is the best possible.

By using Holder's inequality, Beesack [4] gave a refinement of Opial-type inequalities by establishing the conditions under which the weighted Opial-type of (1.2) holds

$$
\left(\int_{a}^{b}|l(x)|^{q}\left|l^{\prime}(x)\right|^{s} v(x) \mathrm{d} x\right)^{\frac{1}{s+q}} \leq C\left(\int_{a}^{b}\left|l^{\prime}(x)\right|^{p} u(x) \mathrm{d} x\right)^{\frac{1}{p}}
$$

for all $l$ with $l(a)=0$, where $p>1, q>0$ and $0<s<p$. The case of negative values of $p, q$ and $s$ was also considered.

One of the special cases of (1.7) when $q=s=u(t)=v(x)=1$ and $p=2$ is:

$$
\int_{0}^{b}|l(x)|\left|l^{\prime}(x)\right| \mathrm{d} x \leq \frac{b}{2} \int_{0}^{b}\left|l^{\prime}(x)\right|^{2} \mathrm{~d} x
$$

and the weighted Hardy inequality is

$$
\left(\int_{a}^{b}\left|\int_{a}^{x} f(t) \mathrm{d} t\right|^{q} v(x) \mathrm{d} x\right)^{\frac{1}{q}} \leq C\left(\int_{a}^{b}|f(t) \mathrm{d} t|^{p} u(t)\right)^{\frac{1}{p}}
$$

If $s=0$, then (1.7) reduces to weighted Hardy inequality (1.9).

In [5], Imoru and Adeagbo-Sheikh established the following result:

Theorem 1.10. Let $\eta$ be continuous and non-decreasing on $[\alpha, \beta]$,

$0 \leq \alpha \leq \beta<\infty$, with $\eta(x)>0$ for $x>0,1 \leq \sigma \leq q<\infty$ and $f(x)$ be non-negative Lebesgue-Stieltjes integrable with respect to $\eta(x)$ on $[\alpha, \beta]$. If $\delta$ is a real number such that $-\frac{\sigma}{q} \leq \delta \leq 0$, then

$$
\begin{aligned}
& {\left[\int_{\alpha}^{\beta} \eta^{\frac{\delta q}{\sigma}}(x)\left(\int_{\beta}^{x} f(t) \mathrm{d} \eta(t)\right)^{q} \mathrm{~d} \eta(x)\right]^{\frac{1}{q}}} \\
& \leq C(\alpha, \beta, \sigma, q, \delta)\left[\int_{\alpha}^{\beta} \eta^{\delta \sigma-1}(x)[\eta(x) f(x)]^{\sigma} \mathrm{d} \eta(x)\right]^{\frac{1}{\sigma}}
\end{aligned}
$$

where

$$
\begin{aligned}
& C(\alpha, \beta, \sigma, q, \delta) \\
& =(-\delta)^{\frac{q(1-\sigma)}{\sigma}}\left(\frac{\sigma}{\sigma+\delta q}\right)^{\frac{\sigma}{q}} \eta(\alpha)^{\sigma+\delta q}\left(\eta(\beta)^{-\delta}-\eta(\alpha)^{-\delta}\right)^{\frac{q(\sigma-1)}{\sigma}}>0
\end{aligned}
$$


Oguntuase [6] presented an integral inequality by using Hölder's inequality to obtain an integral inequality that has Opial's and Hardy's inequalities as special cases. However, he observed that constant (1.12) at the right hand side was wrongly written and obtained a better constant as stated below:

Theorem 1.13. Let $\eta$ be continuous and non-decreasing on $[\alpha, \beta]$, $0 \leq \alpha \leq \beta<\infty$, with $\eta(x)>0$ for $x>0$. Let $q \geq \sigma \geq 1$ and $f(x)$ be non-negative and Lebesgue-Stieltjes integrable with respect to $g(x)$ on $[\alpha, \beta]$.

Suppose $\delta$ is a real number such that $-\frac{\sigma}{q}<\delta<0$ then

$$
\begin{aligned}
& {\left[\int_{\alpha}^{\beta} \eta(x)^{\frac{\delta q}{\sigma}}\left(\int_{\alpha}^{x} f(t) \mathrm{d} \eta(t)\right)^{q} \mathrm{~d} \eta(x)\right]^{\frac{1}{q}}} \\
& \leq C(\alpha, \beta, \sigma, q, \delta)\left[\int_{\alpha}^{\beta} \eta(x)^{(\sigma-1)(1+\delta)} f(x)^{\sigma} \mathrm{d} \eta(x)\right]^{\frac{1}{\sigma}}
\end{aligned}
$$

where

$$
\begin{aligned}
& C(\alpha, \beta, \sigma, q, \delta) \\
& =(-\delta)^{\frac{q(1-\sigma)}{\sigma}}\left(\frac{\sigma}{\sigma+\delta q}\right)^{\frac{1}{q}} g(\alpha)^{\sigma+\delta q}\left(\eta(\beta)^{-\delta}-\eta(\alpha)^{-\delta}\right)^{\frac{q}{\sigma}(\sigma-1)}>0
\end{aligned}
$$

In [7], Adeagbo-Sheikh and Fabelurin follow the trend by using Jensen's and Minkowski's integral inequalities to establish a result that generalized (1.14) as follows:

Theorem 1.16. Let $g$ be a continuous function and non-decreasing on $[a, b]$, $0 \leq a \leq b<\infty$, with $g(x)>0$ for $x>0$. Suppose that $p \geq q \geq 1$, $0<q+s \leq p, \delta>0$ and $f(x)$ is non-negative, non-decreasing and Lebesgue-Stieltjes integrable with respect to $g(x)$ on $[a, b]$. Then

$$
\begin{aligned}
& {\left[\int_{a}^{b}\left[\int_{x}^{b} f(t) \mathrm{d} g(t)\right]^{q} f(x)^{s} v_{1}(x) \mathrm{d} g(x)+B(p, q, s, \delta, g(a))\right]^{\frac{1}{q+s}}} \\
& \leq C(p, q, s, \delta)^{\frac{1}{q+s}}\left[\int_{a}^{b} f(t)^{p} u_{1}(t) \mathrm{d} g(t)\right]^{\frac{1}{p}}
\end{aligned}
$$

where

$$
\begin{aligned}
& v_{1}(x)=g(x)^{\frac{\delta(q+s)}{p}-1}\left[g(x)^{-\delta}-g(b)^{-\delta}\right]^{\frac{(q+s)-p q}{p}}, \\
& u_{1}(t)=g(t)^{\frac{p q(1+\delta)}{q+s}-1}, \\
& C(p, q, s, \delta)=\left[\delta^{-1}\right]^{\frac{p q-(q+s)+p}{p}}\left[\frac{p}{q+s}\right] \text { and } \\
& B(p, q, s, \delta, g(a)) \\
& =C(p, q, s, \delta) g(a)^{\frac{\delta(q+s)}{p}}\left[\int_{a}^{b} g(t)^{\frac{p q(1+\delta)}{q+s}-(1+\delta)} f(t)^{p} \mathrm{~d} g(t)\right]^{\frac{q+s}{p}}
\end{aligned}
$$

Throughout what follows, unless otherwise stated, we shall assume $\eta$ to be continuous, non-negative and non-decreasing. Furthermore, $f$ is assumed to be a 
non-negative integrable function with respect to $\chi$.

The inequality on time scale was introduced by Hilger [8] in order to unify discrete and continuous analysis. Hence, a time scale is an arbitrary non empty closed subset of real numbers. Thus, the real numbers, integers, natural numbers and non negative integers are examples of time scales. However, little work has been done on Opial's inequality on time scales.

A time scale $\mathbb{T}$ is an arbitrary nonempty closed subset of the set $\mathbb{R}$ of all real numbers. Let $\mathbb{T}$ be the topology inherits from standard topology on $\mathbb{R}$. For $t \in \mathbb{T}$, if $t<\sup \mathbb{T}$, we define the forward jump operator $\sigma: \mathbb{T} \rightarrow \mathbb{T}$ by

$$
\sigma(t):=\inf \{\tau>t: \tau \in \mathbb{T}\} \in \mathbb{T}
$$

while if $t>\inf \mathbb{T}$, the backward jump operator $\rho: \mathbb{T} \rightarrow \mathbb{T}$ is defined by

$$
\rho(t):=\sup \{\tau<t: \tau \in \mathbb{T}\} \in \mathbb{T}
$$

If $\sigma(t)>t$, we say $t$ is right scattered, while if $\sigma(t)<t$, we say $t$ is left scattered.

If $\sigma(t)=t$, we say $t$ is right dense, while if $\sigma(t)=t$, we say $t$ is left dense.

Throughout this paper, we let:

a) $\mathbb{R}=(-\infty,+\infty)$;

b) $\mathbb{T}$ is a time scale and;

c) an interval means the intersection of a real interval with the given time scale.

If $f: \mathbb{T} \rightarrow \mathbb{R}$, then $f^{\sigma}: \mathbb{T} \rightarrow \mathbb{R}$ is defined by

$$
f^{\sigma}(t)=f(\sigma(t)), \quad \text { for all } t \in \mathbb{T} .
$$

A mapping $f: \mathbb{T} \rightarrow \mathbb{R}$ is called rd-continuous if it satisfies:

a) $f$ is continuous at each right-dense point or maximal element of $\mathbb{R}$;

b) the left-sided $\lim _{x \rightarrow l^{-}} f(x)=f\left(l^{-}\right)$exists at each left-dense point $t$ of $\mathbb{T}$;

c) an interval means the intersection of a real interval with the given time scale;

d) $C_{r d}(\mathbb{T}, \mathbb{R})=\{f: \mathbb{T} \rightarrow \mathbb{R}$, is an rd-continuous function $\}$

e) $\mathbb{T}^{k}=\left\{\begin{array}{lc}\mathbb{T}-\{m\} & \text { if } \mathbb{T} \text { has a left-scattered maximal point } m \\ \mathbb{T}, & \text { otherwise }\end{array}\right.$;

f) If $f: \mathbb{T} \rightarrow \mathbb{R}$, then $f^{\sigma}: \mathbb{T} \rightarrow \mathbb{R}$ is defined by

$$
f^{\sigma}(t)=f(\sigma(t))
$$

Let $x: \mathbb{T} \rightarrow \mathbb{R}$ and fix $t \in \mathbb{T}^{k}$, then we define $x^{\Delta}(t)$ to be the number (provided it exists) with the property that for any given $\varepsilon>0$, there is a neighborhood $U$ of $t$ such that

$$
\left|[x(\sigma(t))-x(s)]-x^{\Delta}(t)[\sigma(t)-s]\right|<\varepsilon|\sigma(t)-s|,
$$

for all $s \in U$. We call $x^{\Delta}(t)$ the delta derivative of $x(t)$ at $t$. It can be shown that if $x: \mathbb{T} \rightarrow \mathbb{R}$ is continuous at $t \in T$ and $t$ is right scattered, then

$$
x^{\Delta}(t)=\frac{x(\sigma(t))-x(t)}{\sigma(t)-t}
$$


A function $F: \mathbb{T} \rightarrow \mathbb{R}$ is an anti-derivative of $f: \mathbb{T} \rightarrow \mathbb{R}$ if $F^{\Delta}(t)=f(t)$ for all $t \in T^{k}$. In this case, we define the integral of $f$ by

$$
\int_{s}^{t} f(\tau) \Delta(\tau)=F(t)-F(s)
$$

for $s, t \in T^{k}$.

The objective of this paper is to obtain a new integral inequality which is an extension of Theorem 1.1, Theorem 1.3, Theorem 1.5 and Theorem 1.13.

Indeed, we shall show that Theorem 1.1 in its modified form leads to some extensions, variants and a new generalization of a class of inequalities which are related to Hardy's and Opial's integral inequalities. Moreover, we shall examine the case when $\tau=1$.

In fact, some extensions of Hardy's inequality due to Imoru [9] are shown to be immediate consequences of the modified form of Theorem 1.3.

The methodology adopted shall be the following:

Let $[\beta, \alpha] \in \mathbb{T}$ and $\rho, \tau, \gamma, \chi, \gamma, \eta \in \mathbb{T}$. Suppose $\Delta \in \mathbb{C}_{r d}([\beta, \alpha])$ and $\mathbb{R} \in\left([\beta, \alpha]_{\mathbb{T}}, \mathbb{R}\right), \quad \tau \geq 0, \quad \chi \geq 0, \quad \gamma, \eta, \sigma, \varsigma>1, \quad \lambda$ be non-decreasing and $-\infty \leq \alpha \leq \beta<\infty$. Suppose $\phi$ has a continuous inverse $\phi^{-1}$ which is necessarily concave, then

$$
\varphi^{-1}\left(\frac{\int_{\beta}^{\alpha} \rho(\tau, \chi) \Delta \lambda(\chi)}{\int_{\beta}^{\alpha} \Delta \lambda(\chi)}\right) \leq \frac{\int_{\beta}^{\alpha} \varphi^{-1}(\rho(\tau, \chi)) \Delta \lambda(\chi)}{\int_{\beta}^{\alpha} \Delta \lambda(\chi)}
$$

which can be written in the form:

$$
\int_{\beta}^{\alpha} \rho(\tau, \chi)^{\frac{1}{\gamma \eta \sigma \varsigma}} \Delta \lambda(\chi) \leq\left(\int_{\beta}^{\alpha} \Delta \lambda(\chi)\right)^{1-\frac{1}{\eta}}\left(\int_{\beta}^{\alpha} \rho(\tau, \chi)^{\frac{1}{\gamma \eta \varsigma}} \Delta \lambda(\chi)\right)^{\frac{1}{\eta}}
$$

and for $0<\eta<1$. The later inequality is the Jensen's inequality for convex functions and putting convex function $\varphi(t)=t^{\eta}$ in (1.19) yields:

$$
\rho(\tau, \chi)=\kappa(\beta)^{\delta \gamma \sigma \varsigma} \kappa(\chi)^{\tau \gamma \eta \varsigma(1+\delta)} f(\chi)^{\gamma \sigma \varsigma \eta}
$$

and

$$
\lambda \Delta(\chi)=\kappa(\chi)^{-\tau(1+\delta)} \Delta(\chi)
$$

However, the validity of the left hand side of (1.19) solely depends on the right hand side.

\section{Statement of the Main Result}

In this section, we shall show the main result in the following theorems:

Theorem 2.1. Let $[\beta, \alpha] \in \mathbb{T}$ and $\rho, \tau, \gamma, \chi, \gamma, \eta \in \mathbb{T}$. Suppose $\Delta \in \mathbb{C}_{r d}([\beta, \alpha])$ and $\mathbb{R} \in\left([\beta, \alpha]_{\mathbb{T}}, \mathbb{R}\right)$. Let $\kappa(\chi)$ and $f(\chi)$ be absolutely continuous and non-decreasing on $[0, \chi]$ and $0<0 \leq \chi<\infty$ with $\kappa(\chi)>0$ for $\chi>0$ and $\gamma \geq \eta \geq 1, \eta>0, \eta>0$ and $\delta>0 . \Delta \in \mathbb{C}_{r d}([\beta, \alpha])$ and $\mathbb{R} \in\left([\beta, \alpha]_{\mathbb{T}}, \mathbb{R}\right)$ with $\int_{\beta}^{\alpha} \rho(\tau, \chi)^{\frac{1}{\gamma \sigma \eta \zeta}} \mathrm{d} \Delta(\chi)<\infty$ and 


$$
\begin{aligned}
\left(\int_{\beta}^{\alpha} \mathrm{d} \Delta(\chi)\right)^{1-\frac{1}{\eta}}\left(\int_{\beta}^{\alpha} \rho(\tau, \chi)^{\frac{1}{\gamma \sigma \zeta}} \mathrm{d} \Delta(\chi)\right)^{\frac{1}{\eta}}<\infty . \text { If } 0<\eta \leq 1 \text { then we have, } \\
\left(\int_{0}^{\chi} \kappa(\beta)^{\frac{\delta \gamma}{\eta}}\left[\int_{\beta}^{\alpha} f(\chi) \Delta(\chi)\right]^{\gamma} \Delta(\psi)\right)^{\frac{1}{\gamma}} \\
\leq[1-\tau(\delta+1)]^{\frac{1-\eta}{\eta}}\left(\frac{\eta}{\delta \gamma+\eta}\right)^{\frac{1}{\gamma}}\left[\kappa(\alpha)^{\frac{\delta \gamma+\eta}{\eta}}-\kappa(\psi)^{\frac{\delta \gamma+\eta}{\eta}}\right]^{\frac{1}{\gamma}} \\
\times\left[\kappa(\alpha)^{1-\tau(\delta+1)}-\kappa(\beta)^{1-\tau(\delta+1)}\right]^{\frac{\eta-1}{\eta}}\left(\int_{\zeta}^{\chi} \kappa(\chi)^{\frac{\tau(\eta-\sigma)(1+\delta)}{\sigma}} f(\chi)^{\eta} \Delta(\chi)\right)^{\frac{1}{\eta}}
\end{aligned}
$$

whenever $\eta \geq 1$ or $\eta \leq 0$. The inequality is reversed if $0 \leq \eta \leq 1$. The inequality is strict unless either $\eta=1$ or $\kappa=0$.

The constant factor $[1-\tau(\delta+1)]^{\frac{1-\eta}{\eta}}$ is the best possible when the term

$$
\left[\kappa(\alpha)^{\frac{\delta \gamma+\eta}{\eta}}-\kappa(\psi)^{\frac{\delta \gamma+\eta}{\eta}}\right]^{\frac{1}{\gamma}}\left[\kappa(\alpha)^{1-\tau(\delta+1)}-\kappa(\beta)^{1-\tau(\delta+1)}\right]^{\frac{\eta-1}{\eta}}
$$

is valid throughout.

\subsection{Remark}

Let $\chi, \xi \in \mathbb{T}, \xi \leq \chi, \rho \in C_{r d}(\mathbb{T})$ and $\eta=\sigma=1$. By applying Cauchy's inequality with exponent conjugates $\omega$ and $\tau$, we obtain the following results.

$$
\begin{aligned}
& \left(\int_{0}^{\chi} \kappa(\beta)^{\delta \gamma}\left[\int_{\beta}^{\alpha} f(\chi) \Delta(\chi)\right]^{\gamma} \Delta(\psi)\right)^{\frac{1}{\gamma}} \\
& \leq\left(\frac{1}{\delta \gamma+1}\right)^{\frac{1}{\gamma}}\left[\kappa(\alpha)^{\frac{\delta \gamma+1}{1}}-\kappa(\psi)^{\frac{\delta \gamma+1}{1}}\right]^{\frac{1}{\gamma}} \times\left(\int_{\zeta}^{\chi} \omega^{-\frac{1}{\tau}}(\chi) \omega^{\frac{1}{\tau}}(\chi) f(\chi) \Delta(\chi)\right) \\
& =\left(\frac{1}{\delta \gamma+1}\right)^{\frac{1}{\gamma}}\left[\kappa(\alpha)^{\frac{\delta \gamma+1}{1}}-\kappa(\psi)^{\frac{\delta \gamma+1}{1}}\right]^{\frac{1}{\gamma}} \\
& \times\left(\int_{\zeta}^{\chi}\left(\rho^{1-\omega}(\chi) \Delta(\chi)\right)^{\frac{1}{\omega}}\left(\int_{\zeta}^{\chi} \rho^{\frac{1}{\tau}}(\chi) f(\chi) \Delta(\chi)\right)^{\frac{1}{\tau}}\right) \\
& \leq\left(\frac{1}{\delta \gamma+1}\right)^{\frac{1}{\gamma}}\left[\kappa(\alpha)^{\frac{\delta \gamma+1}{1}}-\kappa(\psi)^{\frac{\delta \gamma+1}{1}}\right]^{\frac{1}{\gamma}} \times\left(\int_{\zeta}^{\chi} \rho^{1-\omega}(\chi) \Delta(\chi)\right)^{\frac{1}{\omega}} \\
& \times\left(\int_{\zeta}^{\chi} \rho^{\frac{1}{\tau}}(\chi) f(\chi) \Delta(\chi)\right)
\end{aligned}
$$

\subsection{Remark}

Let $\chi, \xi \in \mathbb{T}, \xi \leq \chi, \rho \in C_{r d}(\mathbb{T})$ and observe that when $\mathbb{T}=\mathbb{R}$, taking $\tau=1$ in (2.3), then (2.3) reduces to Oguntuase's result.

The modified Minkowski integral inequalities (2.5) and (2.6) are stated in Opic and Kufner [10] on page 21 and 22 as follows: 
Lemma 2.4. Let $K(x, y)$ be a non negative measurable function on $(a, b) \times(c, d)$ and let $r \geq 1$ Then,

$$
\left[\int_{a}^{b}\left[\int_{c}^{d} K(x, y) \mathrm{d} y\right]^{r} \mathrm{~d} x\right]^{\frac{1}{r}} \leq \int_{c}^{d}\left[\int_{a}^{b} K^{r}(x, y) \mathrm{d} y\right]^{\frac{11}{r}}
$$

If $K(x, y)=H(x) G(y)$ where $x \in(a, b)$ and $y \in(c, d)$ then (2.5) reduces to

$$
\left[\int_{a}^{b} G(x)\left[\int_{a}^{b} H(y) \mathrm{d} y\right]^{r} \mathrm{~d} x\right]^{\frac{1}{r}} \leq \int_{a}^{b} H(y)\left[\int_{y}^{b} G(x) \mathrm{d} x\right]^{\frac{1}{r}}
$$

The proof of our main results will depend essentially on the following lemmas:

Lemma 2.7. Let $[\beta, \alpha] \in \mathbb{T}$ and $\rho, \tau, \chi, \gamma, \eta \in \mathbb{T}$. Suppose $\Delta \in \mathbb{C}_{r d}([\beta, \alpha])$ and $\mathbb{R} \in\left([\beta, \alpha]_{\mathbb{T}}, \mathbb{R}\right)$ with

$$
\int_{\beta}^{\alpha} \rho(\tau, \chi)^{\frac{1}{\gamma \sigma \eta \zeta}} \mathrm{d} \Delta(\chi)<\infty
$$

which satisfied the following inequality.

$$
\begin{aligned}
& \int_{\beta}^{\alpha}\left(\kappa(\beta)^{\delta \gamma \sigma \varsigma} \kappa(\chi)^{\tau \eta \eta \zeta(1+\delta)} f(\chi)^{\gamma \sigma \varsigma \eta}\right)^{\frac{1}{\gamma \sigma \eta \zeta}} \kappa(\chi)^{-\tau(1+\delta)} \Delta(\chi) \\
& =\int_{\beta}^{\alpha} \kappa(\beta)^{\frac{\delta}{\eta}} \kappa(\chi)^{\tau(1+\delta)} f(\chi) \kappa(\chi)^{-\tau(1+\delta)} \Delta(\chi) \\
& \leq \kappa(\beta)^{\frac{\delta}{\eta}} \int_{\beta}^{\alpha} f(\chi) \Delta(\chi)
\end{aligned}
$$

and the other side of (1.19) implies the following:

Lemma 2.9. Let $[\beta, \alpha] \in \mathbb{T}$ and $\rho, \tau, \gamma, \chi, \gamma, \eta \in \mathbb{T}$. Suppose $\Delta \in \mathbb{C}_{r d}([\beta, \alpha])$ and $\mathbb{R} \in\left([\beta, \alpha]_{\mathbb{T}}, \mathbb{R}\right)$ with

$$
\left(\int_{\beta}^{\alpha} \lambda \Delta(\chi)\right)^{1-\frac{1}{\eta}}\left(\int_{\beta}^{\alpha} \rho(\tau, \chi)^{\frac{1}{\gamma \sigma_{\varsigma}}} \lambda \Delta(\chi)\right)^{\frac{1}{\eta}}<\infty
$$

which also satisfied the inequality.

$$
\begin{aligned}
& \left(\int_{\beta}^{\alpha} \kappa(\chi)^{-\tau(1+\delta)} \Delta(\chi)\right)^{1-\frac{1}{\eta}}\left(\left(\kappa(\beta)^{\delta \gamma \sigma \varsigma} \kappa(\chi)^{\tau \gamma \eta \xi(1+\delta)} f(\chi)^{\gamma \sigma \zeta \eta}\right)^{\frac{1}{\gamma \sigma \zeta}} \kappa(\chi)^{-\tau(1+\delta)} \Delta(\chi)\right)^{\frac{1}{\eta}} \\
& =\left(\int_{\beta}^{\alpha} \kappa(\chi)^{-\tau(1+\delta)} \Delta(\chi)\right)^{1-\frac{1}{\eta}}\left(\int_{\beta}^{\alpha} \kappa(\beta)^{\delta} \kappa(\chi)^{\frac{\eta \tau(1+\delta)}{\sigma}} f(\chi)^{\eta} \kappa(\chi)^{-\tau(1+\delta)} \Delta(\chi)\right)^{\frac{1}{\eta}} \\
& =\left(\int_{\beta}^{\alpha} \kappa(\chi)^{-\tau(1+\delta)} \Delta(\chi)\right)^{1-\frac{1}{\eta}}\left(\int_{\beta}^{\alpha} \kappa(\beta)^{\frac{\delta}{\eta}} \kappa(\chi)^{\frac{\tau(\eta-\sigma)(1+\delta)}{\sigma}} f(\chi)^{\eta} \Delta(\chi)\right)^{\frac{1}{\eta}} \\
& \leq\left(\int_{\beta}^{\alpha} \kappa(\chi)^{-\tau(1+\delta)} \Delta(\chi)\right)^{1-\frac{1}{\eta}} \kappa(\beta)^{\frac{\delta}{\eta}}\left(\int_{\beta}^{\alpha} \kappa(\chi)^{\frac{\tau(\eta-\sigma)(1+\delta)}{\sigma}} f(\chi)^{\eta} \Delta(\chi)\right)^{\frac{1}{\eta}} \\
& =[1-\tau(\delta+1)]^{\frac{1}{\eta}-1}\left[\kappa(\alpha)^{1-\tau(\delta+1)}-\kappa(\beta)^{1-\tau(\delta+1)}\right]^{1-\frac{1}{\eta}} \\
& \times \kappa(\beta)^{\frac{\delta}{\eta}}\left(\int_{\beta}^{\alpha} \kappa(\chi)^{\frac{\tau(\eta-\sigma)(1+\delta)}{\sigma}} f(\chi)^{\eta} \Delta(\chi)\right)^{\frac{1}{\eta}}
\end{aligned}
$$




$$
\begin{aligned}
\leq & {[1-\tau(\delta+1)]^{\frac{\eta-1}{\eta}}\left[\kappa(\alpha)^{1-\tau(\delta+1)}-\kappa(\beta)^{1-\tau(\delta+1)}\right]^{\frac{\eta-1}{\eta}} } \\
& \times \kappa(\beta)^{\frac{\delta}{\eta}}\left(\int_{\beta}^{\alpha} \kappa(\chi)^{\frac{\tau(\eta-\sigma)(1+\delta)}{\sigma}} f(\chi)^{\eta} \Delta(\chi)\right)^{\frac{1}{\eta}}
\end{aligned}
$$

\subsection{Remark}

The Proof of Lemma 2.7 is immediate from the analysis of Lemma 2.9 and hence omitted.

\section{Proof of Theorem}

The proof follows from Lemma 2.7 and Lemma 2.9 by combining (2.8) and (2.10) with further simplification as:

$$
\begin{aligned}
& \kappa(\beta)^{\frac{\delta \gamma}{\eta}}\left[\int_{\beta}^{\alpha} f(\chi) \Delta(\chi)\right]^{\gamma} \\
& \leq[1-\tau(\delta+1)]^{\frac{\gamma(1-\eta)}{\eta}}\left[\kappa(\alpha)^{-1-\tau(\delta+1)}-\kappa(\beta)^{-\delta}\right]^{\frac{\gamma(1-\eta)}{\eta}} \\
& \times \kappa(\beta)^{\frac{\delta \gamma}{\eta}}\left(\int_{\beta}^{\alpha} \kappa(\chi)^{\frac{\tau(\eta-\sigma)(1+\delta)}{\sigma}} f(\chi)^{\eta} \Delta(\chi)\right)^{\frac{\gamma}{\eta}}
\end{aligned}
$$

integrate both sides of $(2.11)$ with respect to $\Delta(\psi)$ from 0 to $\chi$

$$
\begin{aligned}
& \left(\int_{0}^{\chi} \kappa(\beta)^{\frac{\delta \gamma}{\eta}}\left[\int_{\beta}^{\alpha} f(\chi) \Delta(\chi)\right]^{\gamma} \Delta(\psi)\right)^{\frac{\eta}{\gamma}} \\
& \leq[1-\tau(\delta+1)]^{(1-\eta)}\left[\kappa(\alpha)^{(1-\tau(\delta+1))}-\kappa(\beta)^{(1-\tau(\delta+1))}\right]^{\frac{\gamma(\eta-1)}{\eta}} \kappa(\beta)^{\frac{\delta \gamma}{\eta}} \\
& \times\left[\int_{0}^{\chi}\left(\int_{\beta}^{\alpha} \kappa(\chi)^{\frac{\tau(\eta-\sigma)(1+\delta)}{\sigma}} f(\chi)^{\eta} \Delta(\chi)\right)^{\frac{\gamma}{\gamma}} \Delta(\psi)\right]^{\frac{\gamma}{\gamma}}
\end{aligned}
$$

combining (2.6), (2.8) and (2.10) we have the following inequality:

$$
\begin{aligned}
& \left(\int_{0}^{\chi} \kappa(\beta)^{\frac{\delta \gamma}{\eta}}\left[\int_{\beta}^{\alpha} f(\chi) \Delta(\chi)\right]^{\gamma} \Delta(\psi)\right)^{\frac{\eta}{\gamma}} \\
& \leq[1-\tau(\delta+1)]^{1-\eta} \int_{\zeta}^{\chi} \kappa(\chi)^{\frac{\tau(\eta-\sigma)(1+\delta)}{\sigma}} f(\chi)^{\eta} \\
& \times\left[\int_{\beta}^{\alpha}\left[\kappa(\alpha)^{(1-\tau(\delta+1))}-\kappa(\beta)^{(1-\tau(\delta+1))}\right]^{\frac{\gamma(\eta-1)}{\eta}} \kappa(\beta)^{\frac{\delta \gamma}{\eta}} \Delta(\psi)\right)^{\frac{\gamma}{\eta}} \Delta(\chi) \\
& =[1-\tau(\delta+1)]^{1-\eta}\left[\kappa(\alpha)^{(1-\tau(\delta+1))}-\kappa(\beta)^{(1-\tau(\delta+1))}\right]^{(\eta-1)} \\
& \times \int_{\zeta}^{\chi} \kappa(\chi)^{\frac{\tau(\eta-\sigma)(1+\delta)}{\sigma}} f(\chi)^{\eta}\left(\int_{\psi}^{\alpha} \kappa(\beta)^{\frac{\delta \gamma}{\eta}} \Delta(\psi)\right)^{\frac{\gamma}{\eta}} \Delta(\chi) \\
& \leq[1-\tau(\delta+1)]^{1-\eta}\left(\frac{\eta}{\delta \gamma+\eta}\right)^{\frac{\eta}{\gamma}}\left[\kappa(\alpha)^{\frac{\delta \gamma+\eta}{\eta}}-\kappa(\psi)^{\frac{\delta \gamma+\eta}{\eta}}\right]^{\frac{\eta}{\gamma}} \\
& \\
& \times\left[\kappa(\alpha)^{(1-\tau(\delta+1))}-\kappa(\beta)^{(1-\tau(\delta+1))}\right]^{\eta-1} \int_{\zeta}^{\chi} \kappa(\chi)^{\frac{\tau(\eta-\sigma)(1+\delta)}{\sigma}} f(\chi)^{\eta} \Delta(\chi)
\end{aligned}
$$


which implies

$$
\begin{aligned}
& \left(\int_{0}^{\chi} \kappa(\beta)^{\frac{\delta \gamma}{\eta}}\left[\int_{\beta}^{\alpha} f(\chi) \Delta(\chi)\right]^{\gamma} \Delta(\psi)\right)^{\frac{1}{\gamma}} \\
& \leq[1-\tau(\delta+1)]^{\frac{1-\eta}{\eta}}\left(\frac{\eta}{\delta \gamma+\eta}\right)^{\frac{1}{\gamma}}\left[\kappa(\alpha)^{\frac{\delta \gamma+\eta}{\eta}}-\kappa(\psi)^{\frac{\delta \gamma+\eta}{\eta}}\right]^{\frac{1}{\gamma}} \\
& \times\left[\kappa(\alpha)^{1-\tau(\delta+1)}-\kappa(\beta)^{1-\tau(\delta+1)}\right]^{\frac{\eta-1}{\eta}}\left(\int_{\zeta}^{\chi} \kappa(\chi)^{\frac{\tau(\eta-\sigma)(1+\delta)}{\sigma}} f(\chi)^{\eta} \Delta(\chi)\right)^{\frac{1}{\eta}}
\end{aligned}
$$

\subsection{Remark}

Let $\kappa(\beta)=\varsigma, \gamma=\eta, \xi, \chi, \rho \in C_{r d}(\mathbb{T})$. The above inequality becomes

$$
\begin{aligned}
& \left(\int_{0}^{\chi} \varsigma^{\delta}\left[\int_{\beta}^{\alpha} f(\chi) \Delta(\chi)\right] \Delta(\psi)\right) \\
& \leq\left(\frac{1}{\delta+1}\right)\left[\kappa(\alpha)^{\delta+1}-\kappa(\psi)^{\delta+1}\right] \times\left(\int_{\zeta}^{\chi} \varsigma(\chi)^{\frac{\tau(1-\sigma)(1+\delta)}{\sigma}} f(\chi) \Delta(\chi)\right)
\end{aligned}
$$

\section{Discussion of the Results}

We refined some existing results on an integral inequality of Hardy-type on Time-scale and obtained the best possible constant by employing modified Minkowski integral inequalities with some standard lemmas. The results extended and generalized some earlier results in literature.

\section{Conflicts of Interest}

The authors declare no conflicts of interest regarding the publication of this paper.

\section{References}

[1] Opial, Z. (1960) Sur une intégalité. Annales Polonici Mathematici, 8, 29-32. https://doi.org/10.4064/ap-8-1-29-32

[2] Olech, C. (1962) A Simple Proof of a Certain Result of Z. Opial. Annales Polonici Mathematici, 10, 61-63. https://doi.org/10.4064/ap-8-1-61-63

[3] Hardy, G.H. (1959) Note on a Theorem of Hilbert. Mathematische Zeitschrift, 6, 314-317. https://doi.org/10.1007/BF01199965

[4] Beesack, P.R. (1979) Elementary Proofs of Some Opial-Type Integral Inequalities. Journal d Analyse Mathématique, 36, 1-14. https://doi.org/10.1007/BF02798763

[5] Imoru, C.O. and Adeagbo-Sheikh, A.G. (2007) On Some Weighted Mixed Norm Hardy-Type Integral Inequality. Journal of Inequalities in Pure and Applied Mathematics, 8, 5 p.

[6] Oguntuase, J.A. (2009) Remark on an Integral Inequality of the Hardy-Type. Kragujevac Journal of Mathematics, 32, 133-138.

[7] Adeagbo-Sheikh, A.G. and Fabelurin, O.O. (2011) On a Beesack' Inequality Related to Opial's and Hardy's. Kragujevac Journal of Mathematics, 1, 145-150.

[8] Hilger, S. (1998) Analysis on Measure Chains-A Unified Approach to Continuous 
and Discrete Calculus. Results in Mathematics, 18, 18-56.

https://doi.org/10.1007/BF03323153

[9] Imoru, C.O. (1977) On Some Integral Inequalities Related to Hardy's. Canadian Mathematical Bulletin, 20, 307-312. https://doi.org/10.4153/CMB-1977-047-6

[10] Opic, B. and Kufner, A. (1990) Hardy-Type Inequalities, Pitman Research Notes in Mathematics Series. Longman Scientific \& Technical, Harlow, Essex. 